\title{
Hand-Assisted Laparoscopic Surgery: A Versatile Tool for Colorectal Surgeons
}

\author{
Ju Yong Cheong, Christopher J. Young \\ Department of Colorectal Surgery, Royal Prince Alfred Hospital, and University of Sydney, Discipline of Surgery, Sydney, NSW, Australia
}

Purpose: Hand-assisted laparoscopic surgery (HALS) is a minimally invasive surgical technique with the combined benefits of laparoscopic surgery while allowing the use of the surgeon's hand for better tactile control. Obesity has been associated with higher conversion rates with multiport laparoscopic surgery, but not with HALS. This study aimed to examine the versatility of HALS in various clinical contexts.

Methods: All HALSs performed at 2 major tertiary centers in Sydney were prospectively collected for retrospective analysis. Variables including age, sex, body mass index (BMI), previous surgeries, pathologies including size and T-stage, and the number of conversions to a midline laparotomy were examined.

Results: A total of 121 HALS colorectal resections were analyzed. The median age of the patients was 62 years, with $63.6 \%$ being women. Seven patients required conversion to a midline laparotomy. Of the 121 patients, 50.2\% were overweight or obese, and 52.9\% had undergone previous abdominal/pelvic operations. However, neither obesity nor abdominal adhesions from previous operations were an indication for conversion to an open laparotomy in any of the 7 converted patients. The presence of intra-abdominal adhesions did not impact the operative time. HALS allowed access to the entire colon and rectum and allowed resection of the bladder, uterus, and ureter, when these organs were involved.

Conclusion: HALS is a versatile, minimally invasive technique, which is independent of the patient's BMI, for performing a colorectal resection.

Keywords: Hand-assisted laparoscopy; Colorectal surgery

\section{INTRODUCTION}

Hand-assisted laparoscopic surgery (HALS) is a form of minimally invasive laparoscopic surgery [1]. For the colorectal surgeon, it combines the advantages of open (tactile) and laparoscopic (visual magnification) surgery while for the patient, it is minimally invasive (less pain) [2]. HALS has the advantage of allowing manual palpation, which improves depth perception. It also allows manual dissection and better control of hemorrhage should it occur [3]. This has the potential benefit of shortening

Received: March 26, 2017 - Accepted: May 13, 2017

Correspondence to: Christopher J. Young

Royal Prince Alfred Hospital Medical Centre, Suite 415, 100 Carillon Ave, Newtown, NSW, 2042, Australia

Tel: +61-2-9515-7576, Fax: +61-2-9515-1806, E-mail: cyoungnsw@aol.com

(C) 2017 The Korean Society of Coloproctology

This is an open-access article distributed under the terms of the Creative Commons Attribution NonCommercial License (http://creativecommons.org/licenses/by-nc/4.0) which permits unrestricted noncommercial use, distribution, and reproduction in any medium, provided the original work is properly cited. the learning curve associated with laparoscopic surgery [4]. HALS has also been described as being very versatile, allowing the surgeon to more quickly adapt to any variation in the anatomy during colonic surgery, as well as to situations involving dense adhesions, as may be encountered during colonic surgery for inflammatory bowel disease or complex surgeries for diverticular disease with phlegmon $[5,6]$.

One of the public health challenges faced in Western countries is endemic obesity. Operating on obese patients carries inherent risks such as those with associated comorbidities and the higher risk of complications. Obese patients can be technically more challenging, especially when minimally invasive surgery is involved, and higher rates of conversion have been reported for such patients [7]. This study aimed to examine the versatility of HALS in the treatment of patients with colorectal diseases.

\section{METHODS}

Royal Prince Alfred Hospital and Concord Repatriation General 
Hospital are 2 major tertiary centers in Sydney, Australia. Details of all cases of HALS since their introduction (September 2004) have been kept in a database prospectively from the time of operation. After the adoption of HALS techniques, all colorectal cases suitable for a laparoscopic approach were dealt with in either of two ways: (1) all right hemicolectomies with an expected distal transection at the hepatic flexure or proximal transverse colon were completed with a multiport or single incision laparoscopic technique, and (2) all left-sided resections or right-sided resections where the distal transverse resection line was expected to be distal to the midtransverse colon were completed with HALS. Both paper and electronic medical records were reviewed for the patient's demographics, the pathological basis for the surgery, the type of surgery, the need for conversion, and the postoperative outcome. Statistical analyses were performed using IBM SPSS ver. 18.0 (IBM Co., Armonk, NY, USA); P $\leq 0.05$ was chosen as the level of significance.

\section{RESULTS}

Over a 72-month period (1/9/2004 to 7/9/2010), a total of 121

Table 1. Hand-assisted laparoscopic surgery patients' demographics $(\mathrm{n}=121)$

\begin{tabular}{lc}
\hline Demographic & Value \\
\hline Age (yr) & $62(50-73)$ \\
Female sex & $77(63.6)$ \\
Body mass index $\left(\mathrm{kg} / \mathrm{m}^{2}\right)$ & $26.5(23.6-29.7)$ \\
Current smoker & $22(18.2)$ \\
$\quad$ Mean number of packet/year (for smokers) & 40 \\
Alcohol drinkers & $52(43.0)$ \\
Mean number of standard drinks/day (for drinkers) & 2.3 \\
Prior abdominal surgical procedures & \\
0 & $57(47.1)$ \\
1 & $39(32.2)$ \\
2 & $15(12.4)$ \\
3 & $4(3.3)$ \\
4 & $3(2.5)$ \\
5 & $3(2.5)$ \\
ASA PS classification & \\
I & $10(8.3)$ \\
II & $68(56.2)$ \\
II & $29(24.0)$ \\
\hline
\end{tabular}

Values are presented as median (interquartile range) or number (\%) unless otherwise indicated.

ASA PS, American Society of Anesthesiologists physical status.
HALS colectomies were performed. The demographics of those 121 patients are shown in Table 1. The median age of the patients was 62 years, and $63.6 \%$ were women. The age distribution was equal across the genders. The median body mass index (BMI) was $26.5 \mathrm{~kg} / \mathrm{m}^{2}$ (interquartile range, $23.6-29.7 \mathrm{~kg} / \mathrm{m}^{2}$ ), reflecting an overweight cohort. Eighteen percent of the patients were active smokers, and $52.9 \%$ had had at least one prior abdominal or pelvic surgery. The most common pathology requiring HALS operation was a colorectal carcinoma (54.5\%), followed by diverticular disease (16.5\%), then rectal prolapse (9.1\%) (Table 2).

Conversion to a midline laparotomy was required in 7 of the 121 patients (5.7\%) undergoing HALS. The indications for conversion included the patient's inability to tolerate pneumoperitoneum, widespread intraperitoneal endometriosis, a colorectal tumor invading the left ureter and posterior abdominal wall, and involvement of the tumor at the root of the mesentery and the superior mesenteric artery.

Among the cohort of patients, obesity was prevalent. Of the 121 patients, $50.2 \%$ were either overweight or obese, with the maximum BMI of any patient being $39.0 \mathrm{~kg} / \mathrm{m}^{2}, 30.5 \%$ were overweight (BMI, $25-29.9 \mathrm{~kg} / \mathrm{m}^{2}$ ), $12.4 \%$ had class I obesity (BMI, $\left.30-34.9 \mathrm{~kg} / \mathrm{m}^{2}\right), 4.9 \%$ had class II obesity (BMI, $35-39.9 \mathrm{~kg} / \mathrm{m}^{2}$ ), and $2.4 \%$ were morbidly obese (BMI $>40 \mathrm{~kg} / \mathrm{m}^{2}$ or BMI $>35 \mathrm{~kg} /$ $\mathrm{m}^{2}$ with comorbidity). No significant difference in conversion rate was found between patients with normal BMI and overweight patients $(5.5 \%$ vs. $5.4 \%)(\mathrm{P}=0.97)$ or between patients with either grade 1 or grade 2 obesity $(9.5 \%, \mathrm{P}=0.62)$. The mean BMI among the converted group was $27.4 \mathrm{~kg} / \mathrm{m}^{2}$ and that among the nonconverted group was $27.0 \mathrm{~kg} / \mathrm{m}^{2}$, but this difference was not statistically significant $(\mathrm{P}=0.818)$.

Of the 121 patients, $52.9 \%$ had undergone previous abdominal or pelvic operations, with $8.1 \%$ having had three or more operations in the past. Thirty-seven patients (30.6\%) were found to have

Table 2. Hand-assisted laparoscopic surgery patients' operative pathologies $(\mathrm{n}=121)$

\begin{tabular}{lc}
\hline Pathology & Number (\%) \\
\hline Colorectal cancer & $66(54.5)$ \\
Diverticular disease & $20(16.5)$ \\
Rectal prolapse & $11(9.1)$ \\
Tubulovillous adenoma & $10(8.3)$ \\
Ulcerative colitis & $3(2.5)$ \\
No abnormality detected on pathology & $3(2.5)$ \\
Endometriosis & $2(1.7)$ \\
Anal squamous cell cancer & $1(0.8)$ \\
Hyperplastic polyposis & $1(0.8)$ \\
Enterocutaneous fistula & $1(0.8)$ \\
Reversal of Hartmann's procedure & $1(0.8)$ \\
latrogenic bowel injury & $1(0.8)$ \\
\hline
\end{tabular}


extensive adhesions during the operation. However, a previous abdominal/pelvic operation was not an indicator for conversion to a laparotomy in any of the patients. Moreover, the presence of adhesions did not impact the operative time; i.e., no difference in operative time was found between the patients with adhesions and those with no adhesions (281 minutes vs. 268 minutes, $\mathrm{P}=$ 0.45).

Over half of the HALSs (54.5\%) were performed on patients with colorectal cancer (Table 2). The mean diameter of the colon tumors resected by using HALS was $4.0 \mathrm{~cm}$. Oncological integrity was preserved in all patients undergoing HALS for colorectal cancer, with the distal radial margin being at least $50 \mathrm{~mm}$. The mean diameter of the tumors was larger in the converted group $(5.7 \mathrm{~cm})$ than it was in the nonconverted group, and this difference was statistically significant $(3.8 \mathrm{~cm})(\mathrm{P}=0.049)$. The median T-stage for both converted and nonconverted cases was T3, but this difference was not statistically significant $(\mathrm{P}=0.51)$.

The operations performed on the 121 patients by using HALS are shown in Table 3. HALS allowed the entire colon and rectum

Table 3. Hand-assisted laparoscopic surgery procedures performed on 121 patients

\begin{tabular}{lc}
\hline Operation performed & Number \\
\hline High anterior resection (total) & 52 \\
\hline Plus Loop ileostomy & 1 \\
\hline Plus small bowel resection, ileostomy & 1 \\
Plus rectopexy & 8 \\
Low anterior resection (total) & 15 \\
\hline Plus rectopexy & 2 \\
\hline Plus Loop ileostomy & 6 \\
\hline Plus loop ileostomy, appendicectomy & 1 \\
\hline Plus loop ileostomy, repair of femoral hernia & 1 \\
\hline Plus total abdominal hysterectomy, ileocaecal resection, & 1 \\
Abcarian ileostomy & \\
ULAR, J-pouch, loop ileostomy & 4 \\
ULAR, J-pouch, loop ileostomy, partial cystectomy, \\
partial excision of right ureter \\
Right hemicolectomy \\
Hartmann's procedure & 1 \\
Reversal of Hartmann's procedure & 10 \\
Abdominal rectopexy & 3 \\
Proctocolectomy, ileoanal pouch anastomosis, loop & 2 \\
ileostomy & 1 \\
Redo ileocolic resection (previous right hemicolectomy) & 1 \\
Total abdominal colectomy, end ileostomy & 1 \\
\hline Total abdominal colectomy, ileorectal anastomosis & 3 \\
\hline Abdomino-perineal resection & 1 \\
\hline
\end{tabular}

ULAR, ultralow anterior resection. to be accessed for the resection, which ranged from a right hemicolectomy to a total proctocolectomy. HALS was used for both benign and malignant pathologies and was sufficiently versatile so that it could be used in a hysterectomy and a cystectomy, as well as ureter surgery, when indicated by the pathology, without the need for an open laparotomy.

\section{DISCUSSION}

In this study, only $5.7 \%$ of the patients undergoing HALS colorectal resections required conversion to open midline procedures. The conversion rate of HALS was not affected by the patient's obesity, American Society of Anesthesiologists physical status (ASA PS) classification, or previous abdominal or pelvic surgeries. However, key challenges associated with laparoscopic colorectal surgeries are reduced depth perception, poor hand-eye coordination, limited motion, and loss of tactile feedback. Which will be discussed below [8].

The reduction in depth perception increases the risk of potentially dangerous past-pointing errors, which can result in unnecessary tissue damage [9]. Methods to enhance monocular visual cues include using high definition cameras [10], digital processing [11], reintroduction of missing cues such as shadows in the operative field [12], and 3-dimensional (3D) laparoscopic cameras that project the inputs from 2 cameras onto a single monitor. Even with the advent of 3D image-producing laparoscopes, evidence supporting their use is contradictory. A randomized controlled trial comparing 2-dimensional (2D) and 3D laparoscopes showed that a 3D video system did not necessarily improve the efficiency and the quality of surgical techniques or reduce the time required to perform the surgery. In fact, 3D laparoscopy was associated with a higher incidence of visual strain, headache, and facial discomfort [13]. Nevertheless, an ex vivo study found that 3D systems, when compared with 2D systems, resulted in significantly fewer missed grasps and less loss of working material [14].

Compromised hand-eye coordination is also a challenge faced by the surgeon during laparoscopic colorectal surgeries. Key factors contributing to this compromise are the location of the monitor, variable amplification, movement of the mirror, and misorientation [15]. Furthermore, in laparoscopic surgery, the trocar restricts movement by acting as an invariant point. The range of motion is, therefore, reduced to 4 degrees of freedom compared to the 6 needed to perform free motion, thus negatively affecting the surgeon's dexterities [8]. Moreover, the surgeon is not able to directly palpate the organs. Unlike conventional open laparotomies, the completely laparoscopic surgeon cannot use his/her hand/fingertips to assess masses and organs, localize vessels, perform atraumatic retractions, control bleeding, localize tissue planes, or dissect along those planes [8].

Under normal circumstances, redundancy in the human perceptual modalities enables the surgeon to compensate for inadequacies in one modality with cues from other modalities. How- 
ever, in the case of laparoscopic surgery, both visual and tactile perceptions are impaired, resulting in an overall less accurate estimate when visual and tactile cues are integrated. This limitation in visual and tactile perception may subsequently lead to increased cognitive and physical stress among surgeons and potentially to less accurate judgments and estimates [8].

Laparoscopic surgery does have many advantages over open colorectal surgery. A Cochrane Collaboration in 2008 found that laparoscopic surgery, when compared with open colorectal surgeries, was associated with less blood loss, less postoperative pain, improved respiratory function and bowel function, and reduced length of hospital stay [16]. However, laparoscopic surgery is technically more challenging, and the learning curve is considered steeper. Ballantyne [17] reported over 20 years ago in 1995 a rate of conversion to open operation averaging about $25 \%$ during a surgeon's first 50 laparoscopic colectomies. Mackenzie et al. [7] in 2015 developed a risk prediction model for laparoscopic colorectal surgeries for trainees. They found significant risk factors for conversion to be BMI, ASA PS classification, male sex, and prior abdominal surgery, with left hemicolectomy, high anterior resection and low anterior resection conversions being more frequent than right hemicolectomy conversions [7].

HALS is a versatile method that can be performed even in patients with morbid obesity. Nam et al. [18] reported in 2013 on the benefits of using HALS in performing a sigmoid colon cancer resection over open surgery, as did Heneghan et al. [19] in 2013 in a nonrandomized study. The overweight and obese populations are rising in Australia, more so in males [20]. In this study, the overall overweight and obesity rates of $30.5 \%$ and $19.7 \%$, respectively, appear more commensurate with the rates for males in the age range from 60 to 69 years, yet $63 \%$ of the 121 cases in this study involved women, indicating that our cohort was more overweight and obese than the average Australian population [20].

In our cohort, $52.9 \%$ of the patients had undergone previous abdominal or pelvic operation, but the difference in the number of operations between the converted and the nonconverted groups was not statistically significant. This was in the context of operations being performed in a tertiary teaching university hospital by colorectal trainees under supervision. Thus, HALS, with its added benefits of tactile feedback and better depth perception, may be a better tool for trainees to learn than a laparotomy $[3,4]$. This may also be associated with the lower risk of conversion and better patient outcome. The surgeon's intracorporeal hand has the benefit of being able to provide tactile feedback, better organ retraction, dissection, and better control of bleeding, as needed [3]. With the hand placed intra-abdominally, the surgeon has additional information about the depth from proprioceptive feedback gained from the inserted hand. This may shorten the learning curve associated with laparoscopic surgery [1].

Another technical advantage described is that HALS can be used to complete the majority of colectomies without the need for an experienced first assistant. Even complex cases can be man- aged without the need for a second assistant, who otherwise would be needed to complete a straight laparoscopic colorectal resection by utilizing 5 or 6 ports [4]. Moreover, the hand-assisted laparoscopic technique seems to be especially suited to the total colectomy because it allows the surgeon to adapt to the variable anatomy of the transverse mesocolon and the variable adherence of the omentum to the colon and mesocolon, as is often seen with Crohn disease [5]. Furthermore, Aalbers et al. [6] reported that HALS is particularly suitable for the surgical treatment of diverticulitis, where the presence of an inflammatory mass might preclude a successful laparoscopic-assisted approach. Using HALS, the surgeon should be able to manage the diverticular phlegmon more easily and remove it from the pelvis by using finger traction. Limitations of this study include the relatively small number of cases over the time period, the small number of conversions, and the lack of a comparison with a laparoscopic multiport technique group. Nevertheless, this study provides evidence supporting HALS being a versatile tool that may be utilized by colorectal surgeons to face various clinical challenges, including obesity in patients, intra-abdominal adhesions, and bulky disease, whilst arming surgeons with valuable input from tactile feedback.

\section{CONFLICT OF INTEREST}

No potential conflict of interest relevant to this article was reported.

\section{ACKNOWLEDGMENTS}

This study was funded from departmental sources (Department of Colorectal Surgery, Royal Prince Alfred Hospital, Sydney, New South Wales, Australia).

\section{REFERENCES}

1. Moloo H, Haggar F, Coyle D, Hutton B, Duhaime S, Mamazza J, et al. Hand assisted laparoscopic surgery versus conventional laparoscopy for colorectal surgery. Cochrane Database Syst Rev 2010; (10):CD006585.

2. Kim H. Should hand-assisted laparoscopic surgery be placed in the realm of minimally invasive surgery? Ann Coloproctol 2013; 29:42-3.

3. Schadde E, Smith D, Alkoraishi AS, Begos DG. Hand-assisted laparoscopic colorectal surgery (HALS) at a community hospital: a prospective analysis of 104 consecutive cases. Surg Endosc 2006; 20:1077-82.

4. Darzi A. Hand-assisted laparoscopic colorectal surgery. Semin Laparosc Surg 2001;8:153-60.

5. Marcello PW, Fleshman JW, Milsom JW, Read TE, Arnell TD, Birnbaum EH, et al. Hand-assisted laparoscopic vs. laparoscopic colorectal surgery: a multicenter, prospective, randomized trial. Dis Colon Rectum 2008;51:818-26. 
6. Aalbers AG, Biere SS, van Berge Henegouwen MI, Bemelman WA. Hand-assisted or laparoscopic-assisted approach in colorectal surgery: a systematic review and meta-analysis. Surg Endosc 2008;22:1769-80.

7. Mackenzie H, Miskovic D, Ni M, Tan WS, Keller DS, Tang CL, et al. Risk prediction score in laparoscopic colorectal surgery training: experience from the English National Training Program. Ann Surg 2015;261:338-44.

8. Tendick F, Jennings RW, Tharp G, Stark L. Sensing and manipulation problems in endoscopic surgery: experiment, analysis, and observation. Presence 1993;2:66-81.

9. Taffinder N, Smith SG, Huber J, Russell RC, Darzi A. The effect of a second-generation 3D endoscope on the laparoscopic precision of novices and experienced surgeons. Surg Endosc 1999;13: 1087-92.

10. van Bergen P, Kunert W, Schurr MO, Buess GF. Comparative study of endoscopic 2-D and 3-D imaging systems. Langenbecks Arch Chir Suppl Kongressbd 1996;113:634-7.

11. Wenzl R, Lehner R, Holzer A, Windberger U, Heinzl H, Losert UM. Improved laparoscopic operating techniques using a digital enhancement video system. J Am Assoc Gynecol Laparosc 1998; 5:175-8.

12. Mishra RK, Hanna GB, Brown SI, Cuschieri A. Optimum shadow-casting illumination for endoscopic task performance. Arch Surg 2004;139:889-92.

13. Hanna GB, Shimi SM, Cuschieri A. Task performance in endo- scopic surgery is influenced by location of the image display. Ann Surg 1998;227:481-4.

14. Honeck P, Wendt-Nordahl G, Rassweiler J, Knoll T. Three-dimensional laparoscopic imaging improves surgical performance on standardized ex-vivo laparoscopic tasks. J Endourol 2012;26: 1085-8.

15. Eye-hand coordination in laparoscopy: an overview of experiments and supporting aids. Minim Invasive Ther Allied Technol 2001;10:155-62.

16. Schwenk W, Haase O, Neudecker J, Müller JM. Short term benefits for laparoscopic colorectal resection. Cochrane Database Syst Rev 2005;(3):CD003145.

17. Ballantyne GH. Laparoscopic-assisted colorectal surgery: review of results in 752 patients. Gastroenterologist 1995;3:75-89.

18. Nam SE, Jung EJ, Ryu CG, Paik JH, Hwang DY. Feasibility of hand-assisted laparoscopic surgery as compared to open surgery for sigmoid colon cancer: a case-controlled study. Ann Coloproctol 2013;29:17-21.

19. Heneghan HM, Martin ST, Kiran RP, Khoury W, Stocchi L, Remzi $\mathrm{FH}$, et al. Laparoscopic colorectal surgery for obese patients: decreased conversions with the hand-assisted technique. J Gastrointest Surg 2013;17:548-54.

20. Hayes AJ, Lung TW, Bauman A, Howard K. Modelling obesity trends in Australia: unravelling the past and predicting the future. Int J Obes (Lond) 2017;41:178-85. 